\title{
Preparation and Characterization of Anthocyanin Dye and Counter Electrode Thin Film with Carbon Nanotubes for Dye-Sensitized Solar Cells
}

\author{
Ho Chang ${ }^{1, *}$, Kun-Ching $\mathrm{Cho}^{2}$, Tien-Li Chen ${ }^{3}$, Kung-Hui Chu ${ }^{2}$ and Lii-Jenq Jiang ${ }^{1}$ \\ ${ }^{1}$ Department of Mechanical Engineering, National Taipei University of Technology, Taipei 10608, Taiwan, R. O. China \\ ${ }^{2}$ Department of Civil Engineering, Texas A\&M University, WERC 205G,3136, TAMU, TX,77843-3136, USA \\ ${ }^{3}$ Department of Industrial Design, National Taipei University of Technology, Taipei 10608, Taiwan, R. O. China
}

This study mainly explores the incorporation of counter electrode thin film and extracted natural anthocyanin dye in dye-sensitized solar cells (DSSCs). We adopt single-walled carbon nanotubes (SCNTs), with diameters of 1-2 nm and lengths of 1-20 $\mu$ m, as well as multi-walled carbon nanotubes (MCNTs), with diameters of 10-30 nm and lengths of 10-15 $\mu \mathrm{m}$. This study also uses a doctor bade to coat the purified carbon nanotubes on fluorine-doped tin oxide (FTO) conductive glass, so as to form thin film with single layer thickness of around $100 \mu \mathrm{m}$. In addition, for preparation of natural dye, anthocyanin dye is extracted from mulberry fruits. The experimental results show that the DSSCs which are incorporated with purified SCNT counter electrode thin film and anthocyanin dye extracted from mulberry fruit can achieve the photoelectric conversion efficiency of $0.672 \%$. [doi:10.2320/matertrans.M2011063]

(Received February 22, 2011; Accepted July 19, 2011; Published September 7, 2011)

Keywords: dye-sensitized solar cells (DSSCs), carbon nanotubes (CNTs), natural dye, counter electrode, photoelectrode, anthocyanin

\section{Introduction}

A dye-sensitized solar cell (DSSC) is a kind of device that converts visible light into electrical energy. This involves a kind of photosensitization established on a wide-band semiconductor. ${ }^{1)}$ Due to their merit of low cost, DSSCs are have received great attention. The manufacturing process of DSSCs is simple and easy. They can be incorporated in a general environment at room temperature. The cost is only around $1 / 3$ of the manufacturing cost of traditional silicon substrate solar cells. In the future, if the cost of DSSCs can be further reduced, the total cost of DSSCs, when compared with silicon substrate solar cells with the same efficiency, will be only $1 / 10$ of the cost of silicon substrate solar cells. ${ }^{2)}$ In 1991, the Swiss Grätzel research team developed a kind of low-cost, high-efficiency solar cell, with photoelectric conversion efficiency at around $7 \%$. ${ }^{3}$ ) To date, the counter electrodes of DSSCs have primarily been made by expensive metal materials, such as platinum (Pt) and aurum $(\mathrm{Au})$, thus causing the manufacturing costs of DSSCs rise to a great extent. $^{4,5)}$ In 1996, the Swiss Grätzel research team developed a preparation of counter electrode thin film for solar cells. In this preparation process, the thin film is made by mixing graphite, carbon black and $\mathrm{TiO}_{2}$, tremendously reducing the manufacturing costs of DSSCs. In 2006, the Grätzel research team again discovered the use of carbon black to make counter electrode thin film for solar cells, achieving a photoelectric conversion efficiency as high as $9.1 \%$. There seems to be a trend of replacing traditional solar cells made of expensive metallic materials. ${ }^{6}$ In 2008, the research team led by $\mathrm{H}$. Zhu, H. Zeng et al., published a paper about using carbon nanotubes (CNTs) to prepare counter electrodes and anthocyanin dye for application to DSSCs, and the acquired solar energy-to-electricity conversion efficiency was $1.46 \% .^{7)}$ In recent years, CNT thin film has attracted the interests of quite a lot of societies and industries. The unique electronic and structural properties of CNTs have prompted

*Corresponding author, E-mail: f10381@ntut.edu.tw researchers to incorporate them as integrative building blocks for solar cells. ${ }^{8,9)}$ Adding CNTs in DSSCs not only can improve the electrical conductivity of photoelectrode $\left(\mathrm{TiO}_{2}\right.$ film), but also can make the transmission path of photoelectrons smoother, decrease the occurrence of dark current, and reduce the probability of electron-hole recombination. In addition, CNTs can also increase the light scattering nature of DSSCs, and furthermore, enhance the light-harvesting efficiency of DSSCs. Therefore, adding CNTs in DSSCs can enhance the short-circuit photocurrent (Jsc) of DSSCs, thus enhancing both the efficiency of photoelectrode and its lightto-electricity efficiency. The efficiency of cells is mainly determined by the sentizer used. The marketed commercial dyes are mostly chemical compounds, such as N719 and N3 dyes, which both have quite good photoelectric conversion efficiency. However, some expensive metals have to be added to the dye itself, which not only brings about higher manufacturing costs, but also easily leads to environmental pollution. ${ }^{10)}$ In recent years, several breakthrough studies have immensely improved the photoelectric conversion efficiency of DSSCs, thus offering greater chances for carbon counter electrodes and natural dyes to be commonly used in DSSCs in the future. These improvements have also enhanced the photoelectric conversion efficiency of DSSCS, reaching a level that meets civic and industrial needs. ${ }^{1-13)}$ The main reason for this is that nature dye itself possesses the advantages of high usability and extremely low cost. ${ }^{14)}$ This study mainly explores the preparation of counter electrode thin film. A nanotube is taken as the material because nanotubes have good chemical stability and conduction. They do not react to other materials, they perform electronic transfer quickly, they do not create loss of electrons, and they can increase open-circuit voltage and enhance short-circuit current. As for the preparation of the natural dye, dye extracted from mulberry fruits is taken as the photosensitizer.

The study mainly takes CNT as the counter electrode of DSSC, and explores the comparison of conversion efficiency of DSSC before and after purification of SCNTs and MCNTs. In addition, the paper compares the effects of 4 dyes, 
including 3 self-made natural dyes, namely chlorophyll dye, anthocyanin dye and cocktail dye, and the chemical synthetic dye N719, on the characteristics of the open-circuit voltage decay for the prepared DSSCs. The paper also discusses the relationship between the electronic life cycle $\left(\tau_{\mathrm{n}}(\mathrm{s})\right)$ and the open-circuit voltage $\left(V_{\text {oc }}\right)$ for these 4 dyes in DSSCs.

\section{Experimental Details}

The single-walled carbon nanotubes (SCNTs) and multiwalled carbon nanotubes (MCNTs) used in the study have purity of above $95 \%$. CNTs are placed in nitric acid solution to be stirred in thermal reflux at $150^{\circ} \mathrm{C}$ for $5 \mathrm{~h}$. The acid washed solution and distilled water are added to a $2000 \mathrm{ml}$ beaker. After the solution is stirred for one day, it is placed aside and kept still for precipitation, and the water on the upper layer is poured out. After the filtering process, a great deal of distilled water is added to the beaker to wash the nanotubes, making the $\mathrm{pH}$ value of the filtered fluid become neutral. After that, the neutral CNTs are placed in a vacuumed oven for drying. Finally, the solid powder is grinded in an agate mortar to make fine powder for the acquisition of purified CNTs. The forms of purified SCNTs and MCNTs are shown in their FE-SEM photos in Fig. 1. As seen from the figure, the SCNTs have diameters of $1-2 \mathrm{~nm}$ and lengths of $1-20 \mu \mathrm{m}$; and the MCNTs have diameters of $10-30 \mathrm{~nm}$ and lengths of $10-15 \mu \mathrm{m}$.

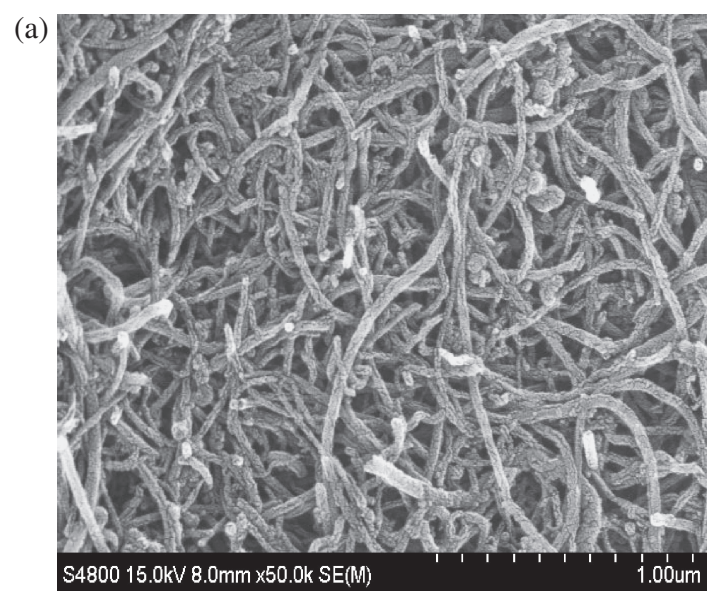

(b)

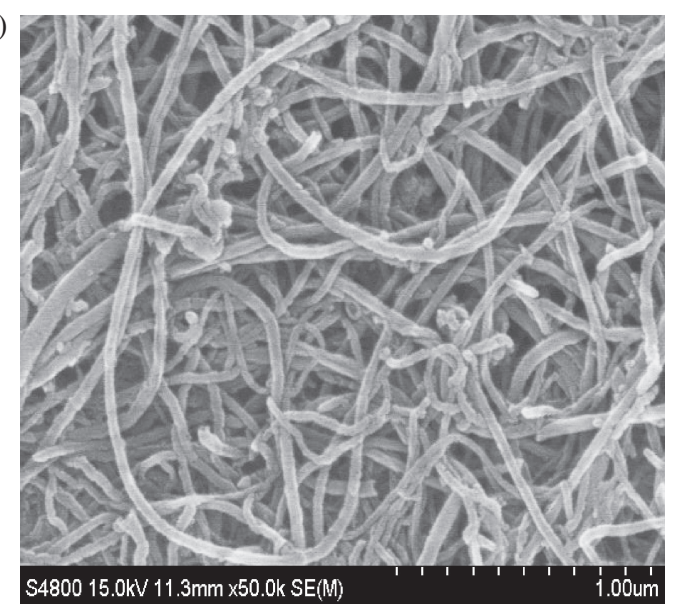

Fig. 1 FE-SEM image of (a) purified SCNTs; and (b) purified MCNTs.
The process used for the preparation of counter electrode thin film conducts coating by using a doctor blade. The 4 carbon materials are unpurified SCNT, purified SCNT, unpurified MCNT and purified SCNT. Each of these 4 CNTs is mixed with acetylacetone, TritonX-100 and $\mathrm{H}_{2} \mathrm{O}$ to form slurry. Material 1: The unpurified SCNT, acetylacetone, TritonX-100 and $\mathrm{H}_{2} \mathrm{O}$ are mixed well in the proportion of $10: 20: 30: 40$ to form a slurry, which is then coated evenly on fluorine-doped tin oxide (FTO) conductive glass by the doctor blade, producing unpurified SCNT counter electrode thin films, with single layer coatings with a thickness of around $100 \mu \mathrm{m}$. Material 2: The purified SCNT, acetylacetone, TritonX-100 and $\mathrm{H}_{2} \mathrm{O}$ are mixed well in the proportion of $10: 10: 10: 25$ to form a slurry, which is then coated evenly on FTO conductive glass by the doctor blade, producing purified SCNT counter electrode thin films, with single layer coatings with a thickness of around $100 \mu \mathrm{m}$. Material 3: The unpurified MCNT, acetylacetone, TritonX100 and $\mathrm{H}_{2} \mathrm{O}$ are mixed well in the proportion of $10: 20$ : $20: 50$ to form a slurry, which is then coated evenly on FTO conductive glass by the doctor blade, producing unpurified MCNT counter electrode thin films, with single layer coatings with a thickness of around $100 \mu \mathrm{m}$. Material 4: The purified MCNT, acetylacetone, TritonX-100 and $\mathrm{H}_{2} \mathrm{O}$ are mixed well in the proportion of $10: 10: 10: 25$ to form a slurry, which is then coated evenly on FTO conductive glass by the doctor blade, producing purified MCNT counter electrode thin films, with single layer coatings with a thickness of around $100 \mu \mathrm{m}$. Thin films have to be additionally sintered under $300^{\circ} \mathrm{C}$ for $1 \mathrm{~h}$ so as to increase the compactness of thin films. The purpose of the above preparation is to explore the effects of the counter electrode thin films of different carbon materials on their photoelectric conversion efficiency.

Put $20 \mathrm{~g}$ of fresh mulberry fruit in $100 \mathrm{ml}$ of a mixed solution containing methanol/water/acetic acid at a proportion of $25: 21: 4$, and heat them all in a double boiler to extract the natural dye. With a heating temperature of $50^{\circ} \mathrm{C}$ and a heating time of $5 \mathrm{~min}$, anthocyanin is extracted. The solid residue in the solution is placed in a filter paper in a clean and light-isolated working environment to filter out and remove the impurities of the dye solution, acquiring a pure, clean and natural dye-anthocyanin solution. A Fourier transform infrared spectrometer (FT-IR) is adopted to analyze the surface functional groups of the prepared natural dye. After the FR-IR test, it is confirmed that for the functional groups of the prepared natural dye, stretching peaks of $\mathrm{O}-\mathrm{H}$ groups with hydrogen-bonded alcohol or phenol occur at $3424 \mathrm{~cm}^{-1}$, stretching peaks of $\mathrm{C}-\mathrm{H}_{3}$ groups occur at $2926 \mathrm{~cm}^{-1}$, stretching peaks of $\mathrm{C}-\mathrm{H}_{2}$ groups occur at $2854 \mathrm{~cm}^{-1}$, stretching peaks of carbon conjugated bond occur at $1629 \mathrm{~cm}^{-1}$, and stretching peaks of C-O groups occur at $1061 \mathrm{~cm}^{-1}$. After comparison with the chemical structure of anthocyanin, it is confirmed that the prepared natural dye has the characteristics of functional groups of anthocyanin.

As for preparation of the photoelectrode thin films, electrophoretic deposition (EPD) is employed. Electrophoretic suspension is made by mixing commercial $\mathrm{TiO}_{2}$ (Degussa P25) with $100 \mathrm{ml}$ of IPA. After that, electrophoretic suspension is placed in a supersonic vibrator and vibrated for $1 \mathrm{~h}$. Then, by EPD at room temperature, a positive-electrode 
aluminum plate and negative-electrode FTO conductive glass are placed in electrophoretic suspension parallel to each other, with a distance of $1 \mathrm{~cm}$ between them. The voltage is set at $40 \mathrm{~V}$, so as to deposit $\mathrm{TiO}_{2}$ photoelectrode thin films with single layers with thicknesses of $15-20 \mu \mathrm{m}$. In addition, thin films have to be additionally sintered at $400^{\circ} \mathrm{C}$ for $1 \mathrm{~h}$ so as to increase the compactness of the thin films. Finally, $\mathrm{TiO}_{2}$ photoelectrode thin films are soaked in the natural dye of mulberry extract for $24 \mathrm{~h}$ in order to make molecules of the natural dye to be absorbed on $\mathrm{TiO}_{2}$ photoelectrode thin films.

Assembly is carried out according to the general assembly procedures of DSSCs. The entire DSSC is composed of FTO conductive glass, $\mathrm{TiO}_{2}$ nanoparticles, natural dye, an electrolyte, a counter electrode and an insulation spacer. When the dye absorption procedures for the photoelectrode are completed, acetonitrile is used to clean the surface of the photoelectrode so as to remove the unabsorbed natural dye on the surface of the $\mathrm{TiO}_{2}$ nanoparticles. After being placed still for drying, the photoelectrode of the DSSC can be obtained. Furthermore, the oxidizing and reducing electrolyte $I^{-} / I_{3}{ }^{-}$is poured in the CNT counter electrode and the gap of the $\mathrm{TiO}_{2}$ photoelectrode. A clip is used for fixing, and to prevent production of air bubbles. As a result, sandwich-structured DSSCs are produced. In a performance test of the prepared DSSCs, the light of a xenon (Xe) lamp with a power of 150$\mathrm{W}$ is selected to simulate sunlight (AM 1.5), and is combined with a Keithley 2400 to form an I-V curve analyzer. Before testing, the distance between the light source and sample should be tested, and the light density should be set to $100 \mathrm{~mW} / \mathrm{cm}^{2}$. The measured results can form an I-V curve. Through the I-V curve, the data of $V_{\mathrm{oc}}(\mathrm{V}), I_{\mathrm{sc}}\left(\mathrm{mA} / \mathrm{cm}^{2}\right)$, FF and $\eta \%$ can be acquired.

\section{Results and Discussion}

Figure 2 shows the XRD spectra of unpurified and purified SCNTs. The data are matched with the database published by the Joint Committee on Powder Diffraction Standards (JCPDS). Figure 2(b) shows that the characteristic peaks of unpurified SCNTs at $2 \theta=26.2^{\circ}, 43.4^{\circ}$ are the diffraction peaks of carbon (corresponding to diffraction planes 002 , 101) after matching with the above database; the characteristic peak at $2 \theta=64.8^{\circ}$ is found to be the diffraction peak of aluminum (corresponding to diffraction plane 220, JCPDS card no. 85-1327) after matching; and the characteristic peak at $2 \theta=44.5^{\circ}$ is found to be the diffraction peak of nickel (corresponding to diffraction plane 111, JCPDS card no. 700989) after matching. As seen in from Fig. 2(a), the characteristic peaks of purified SCNTs are only left with $2 \theta=26.2^{\circ}, 43.4^{\circ}$ (diffraction peaks of carbon), implying that after purification, the only ingredient left in the powder is carbon. In order to verify whether the purified SCNTs have their impurities removed, the study once used an energy dispersive spectrometer (EDS) to make analysis. The test results made by EDS show that after purification of SCNTs, their carbon content increases from the original $87.84 \%$ to $95.35 \%$; their oxygen content decreases from the original $6.43 \%$ to $4.65 \%$; their metal catalysts, nickel (Ni) and aluminum (Al) decrease from the original $4.71 \%$ and $1.02 \%$ to almost $0 \%$.

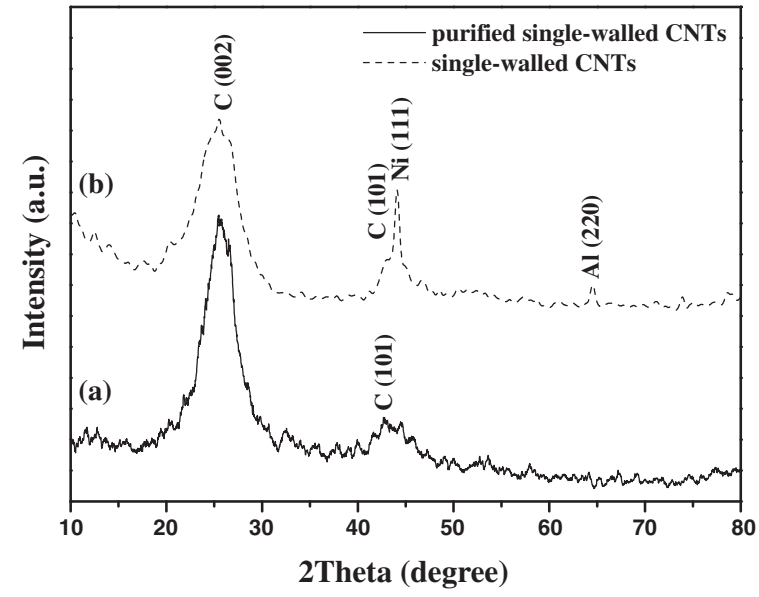

Fig. 2 XRD spectra of unpurified and purified SCNTs.

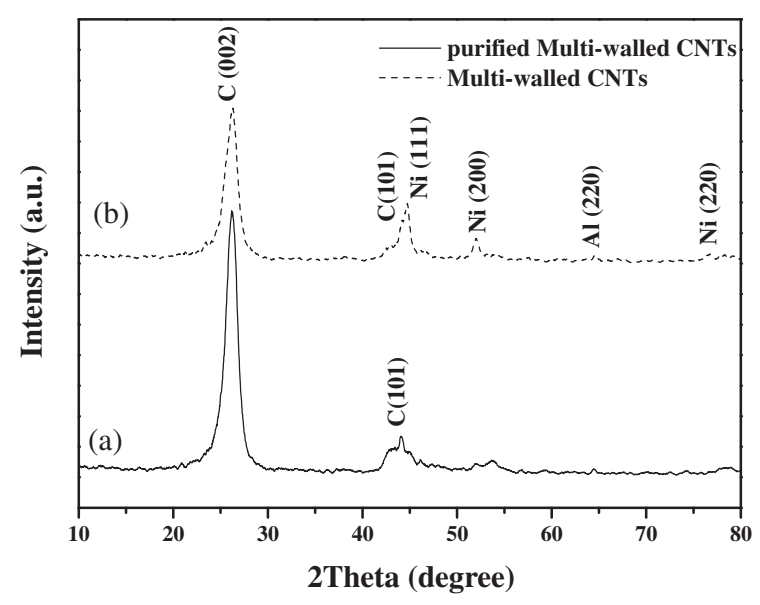

Fig. 3 XRD spectra of unpurified and purified MCNTs.

Figure 3 shows the XRD spectra of unpurified and purified MCNTs. The data are matched with the database published by JCPDS. Figure 3(b) shows that the characteristic peaks of unpurified MCNTs at $2 \theta=26.2^{\circ}, 43.4^{\circ}$ are the diffraction peaks of carbon (corresponding to diffraction planes 002 , 101) after matching with the above database; the characteristic peak at $2 \theta=64.8^{\circ}$ is found to be the diffraction peak of aluminum (corresponding to diffraction plane 220, JCPDS card no. 85-1327) after matching; and the characteristic peaks at $2 \theta=44.5^{\circ}, 52.0^{\circ}, 77.6^{\circ}$ are found to be the diffraction peaks of nickel (corresponding to diffraction planes 111, 200, 220, JCPDS card no. 70-0989) after matching. As seen in Fig. 3(a), the characteristic peaks of purified MCNTs are only left with $2 \theta=26.2^{\circ}, 43.4^{\circ}$ (diffraction peaks of carbon), implying that after purification, the only ingredient left in the powder is carbon. Furthermore, the test results made by EDS show that after purification of MCNTs, their carbon content increases from the original $82.76 \%$ to $95.11 \%$; their oxygen content decreases from the original $9.53 \%$ to $4.89 \%$; their metal catalysts, nickel (Ni) and aluminum $(\mathrm{Al})$ decrease from the original $5.34 \%$ and $2.37 \%$ to almost $0 \%$. As known from the above results, after nitrate purification of CNTs, the metal catalysts and impurities of carbon left in CNTs can be effectively removed to raise the purity of CNTs. 


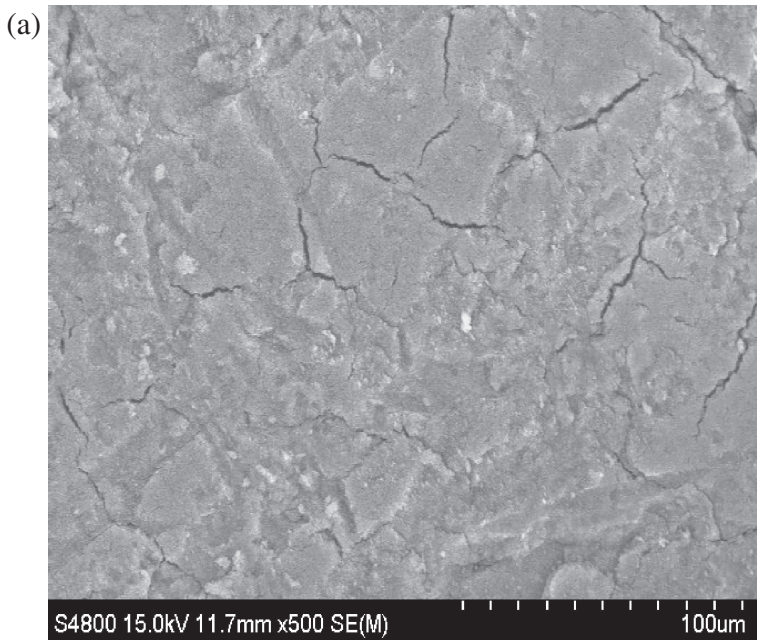

(b)

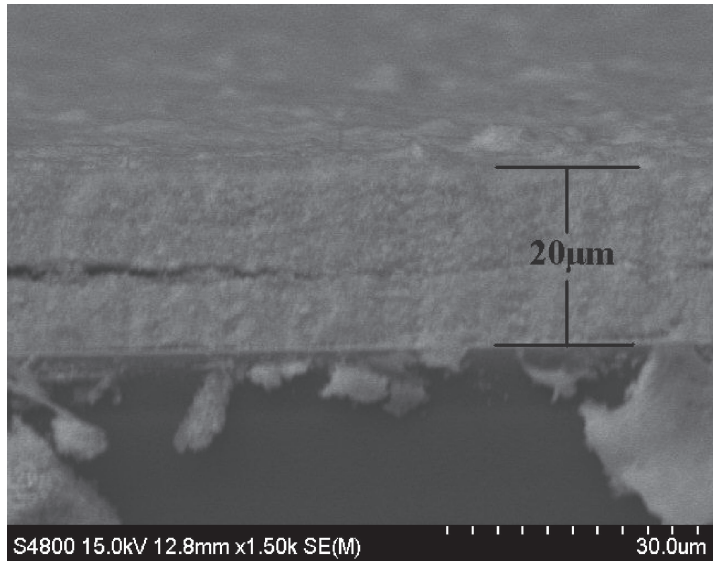

Fig. 4 (a) FE-SEM image; and (b) cross-section image, of Degussa P25 $\mathrm{TiO}_{2}$ thin film.

As shown in Fig. 4(a), the surface of the electrophoretic photoelectrode thin film prepared two times is made of Degussa P25 $\mathrm{TiO}_{2}$, and cracks are found on the thin film surface. The reason for this is that although there are certain favorable conditions, EPD has the shortcoming of producing cracks after the drying of thin film. Hence, the evaporation of the solvent residue in drying process leads to contraction of the thin film layer and the production of cracks. As the drying speed of the thin film is faster, the residue stress caused is greater, and the cracks formed on thin film are more obvious. The electrophoretic solvent selected by this experiment is IPA. IPA is a highly volatile liquid. When the deposited sample is taken out, the IPA at the bottom is rapidly evaporated towards the top, thus leading to the production of cracks on the thin film. Figure 4(b) shows the cross-section image of the thin film. The thickness of its electrophoretic thin film is around $20 \mu \mathrm{m}$.

Figure 5(a) shows the surface of the purified SCNT counter electrode thin film prepared by a doctor blade. It can be seen that some particles exist on the thin film surface. They are actually the particles of chemical substances of the surfactant and ethyl acetone added when the slurry is being grinded. Figure 5(b) shows the cross-section image of the counter electrode thin film after heat treatment at $300^{\circ} \mathrm{C}$. As shown in the figure, the thickness of a single layer of purified SCNT counter electrode thin film is $100 \mu \mathrm{m}$. (a)

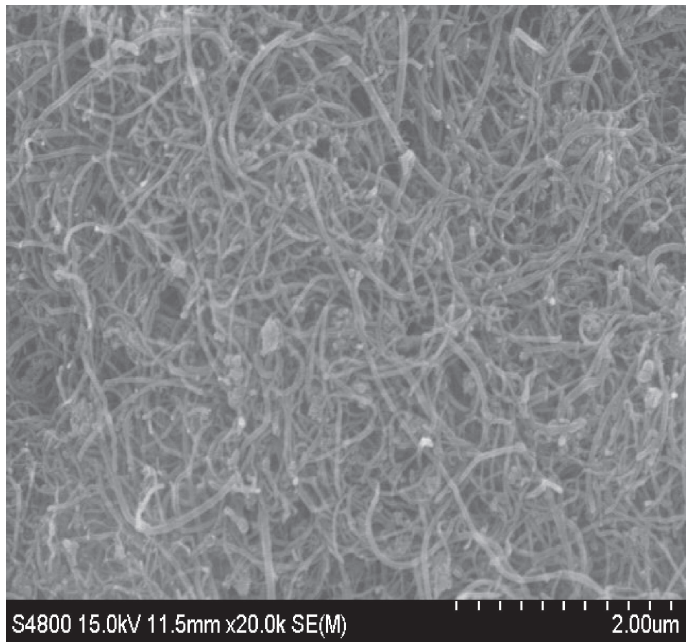

(b)

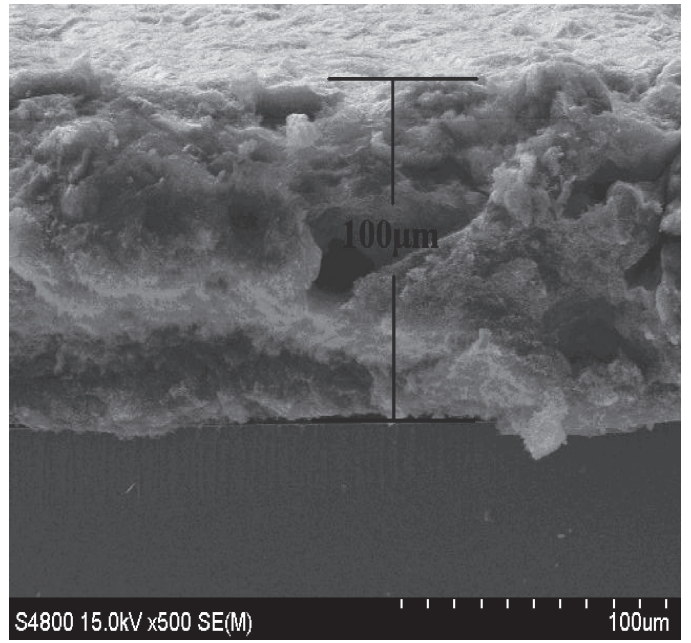

Fig. 5 (a) FE-SEM image; and (b) cross-section image, of purified SCNT thin film.

Figure 6(a) shows the surface of purified MCNT counter electrode thin film prepared by a doctor blade. It can be seen that some particles exist on the thin film surface. They are actually the particles of the chemical substances of the surfactant and ethyl acetone added when slurry is being grinded. Figure 6(b) shows the cross-section image of the counter electrode thin film after heat treatment at $300^{\circ} \mathrm{C}$. As shown in the figure, the thickness of a single layer of purified MCNT counter electrode thin film is around $100 \mu \mathrm{m}$.

Figure 7 shows the incident photon-to-electron conversion efficiency (IPCE) and UV-vis of anthocyanin dye extracted from mulberry fruit. At the zone of $400-700 \mathrm{~nm}$, the photoelectric conversion values allow the light energy to be converted to electrical energy within this wavelength zone. As known from Fig. 7, the IPCE curve and UV-vis pattern of the prepared anthocyanin dye have very similar trends. It refers that when the wavelength is within the range of 400$800 \mathrm{~nm}$, the light absorption degree of the anthocyanin dye can form a similar proportion for transfer to IPCE value. When matching with UV-vis absorption spectrum in the figure, it can be found that the incident photon conversion efficiency $\left(\mathrm{IPCE}_{\max }\right.$ ) at $550 \mathrm{~nm}$ is around $7.2 \%$. Moreover, the maximum incident photon-to-electron conversion efficiency IPCE $_{\max } 400 \mathrm{~nm}$ is around $7.5 \%$. The IPCE and UV- 
(a)

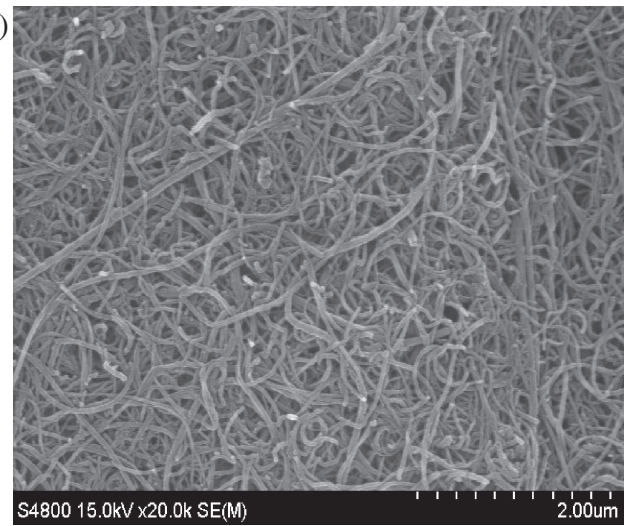

(b)

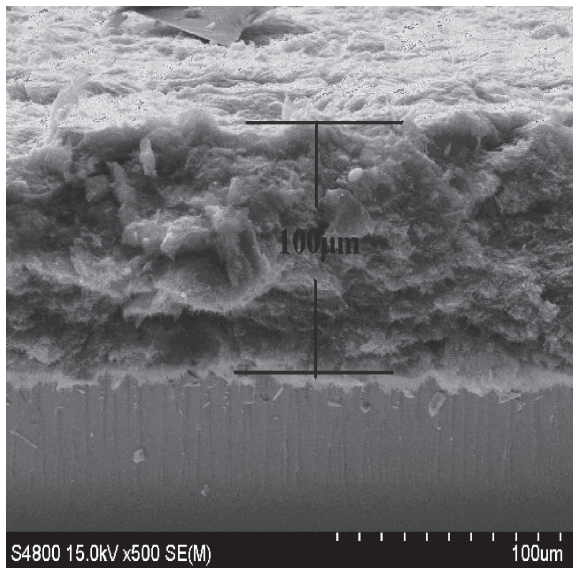

Fig. 6 (a) FE-SEM image; and (b) cross-section image, of purified MCNT thin film.

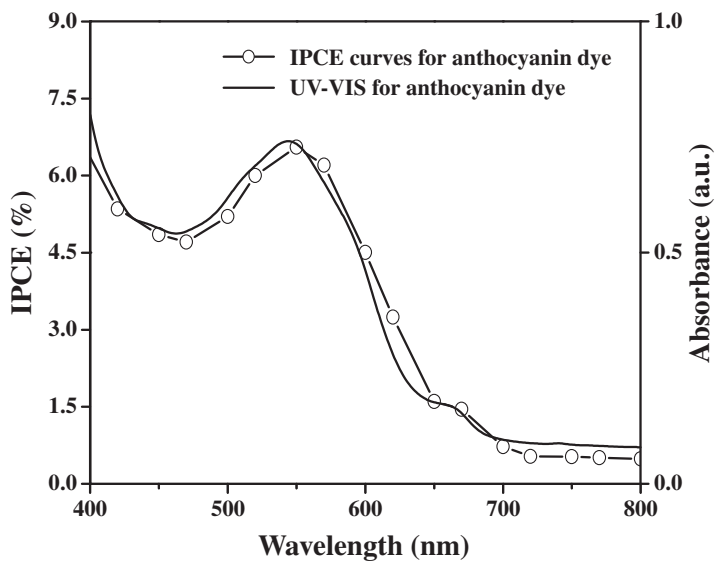

Fig. 7 IPCE curve and absorption spectrum curve of DSSCs sensitized by anthocyanin dye.

vis absorption spectrums of anthocyanin dye show the same trend.

Figure 8 shows the open-circuit voltage $\left(V_{\mathrm{oc}}\right)$-time $(\mathrm{T})$ characteristics curves of different dyes for DSSCs. Of the various dyes, chlorophyll dye is extracted from pomegranate leaves, and cocktail dye is a mixed dye composed of chlorophyll dye and anthocyanin dye at the ratio of $1: 1$. As seen in Fig. 8, when light source is cut off, the open-circuit voltage value of the DSSC absorbed with the N719 dye rapidly falls to zero within $15 \mathrm{~s}$, but that of the DSSC absorbed with natural dye does not fall to zero until $40 \mathrm{~s}$ later.

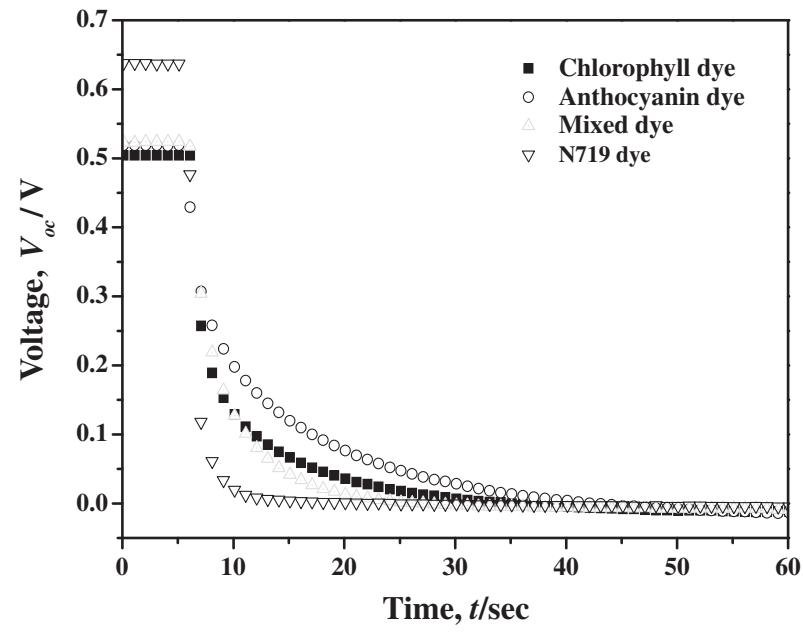

Fig. 8 Chart of open-circuit voltage $\left(V_{\mathrm{oc}}\right)$-time $(T)$ characteristics curves of different dyes.

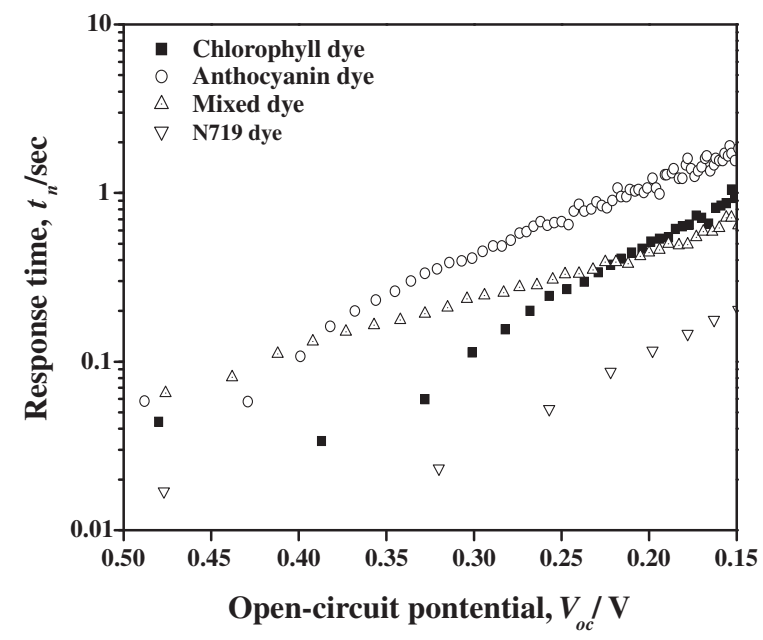

Fig. 9 Curve changes of electronic life cycles $\left[\tau_{\mathrm{n}}(\mathrm{S})\right]$ of different dyes with open-circuit voltage $\left(V_{\mathrm{oc}}\right)$.

After conversion, a relation diagram showing the change of electronic life cycle $\left[\tau_{\mathrm{n}}(\mathrm{s})\right]$ with open-circuit voltage $\left(V_{\mathrm{oc}}\right)$ can be obtained. Figure 9 shows the chart after conversion. After the molecules of chlorophyll dye have absorbed visible light, a large number of conjugated double bonds are produced. This structure, a property of the molecules of chlorophyll dye, is particularly stable because of molecular resonance. As seen in Fig. 9, when the light source is cut off, chlorophyll dye does not excite electrons anymore, and the electrons which are in an excited state earlier are continuously delivered on the $\mathrm{TiO}_{2}$ conduction band. The electrons in the medium-voltage and low-voltage zones are trapped by the trap density of $\mathrm{TiO}_{2}$. In the medium low voltage zone, the electronic life cycle of the anthocyanin dye is longer than that of the chlorophyll dye because anthocyanin dye contains a small amount of $\beta$-carotene, which is a kind of pigment molecule without sensitization properties. After it absorbs light energy, it releases some energy. The energy will be delivered to the electrons being captured earlier, thus causing the captured electrons to return to the $\mathrm{TiO}_{2}$ conduction band again to continue conduction. Therefore, anthocyanin dye has longer electronic life cycle than chlorophyll dye. This implies 


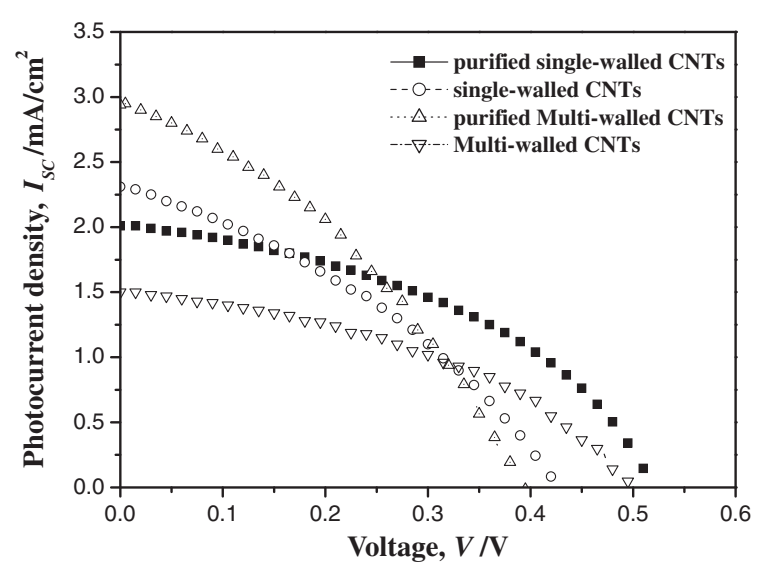

Fig. 10 I-V characteristics of different CNTs for preparation of counter electrode thin films to be incorporated in DSSCs.

that although the dye without sensitization properties cannot play the role of an electron flow supplier in a DSSC module, it can supply energy to electrons when electrons are captured, and make them deliver continuously.

Figure 10 shows the I-V characteristics of different CNTs for the preparation of counter electrode thin films to be incorporated in DSSCs. As seen in Fig. 10, after comparison of the photoelectric conversion efficiencies among the counter electrode thin films prepared by different carbon materials, the purified SCNT counter electrode thin film has higher efficiency, possibly because the purified SCNT counter electrode thin film can effectively deliver electrons, and increase the delivery speed of electrons. As for the inferior efficiency results of the counter electron thin films produced by SCNTs, purified MCNTs and MCNTs, it is possibly that in the delivery process of electrons, electrons are reduced by electrolytes. In addition, the delivery speed of electrons is reduced, thus causing obstacles to the delivery of electrons, and making their photoelectric conversion efficiency poor. Furthermore, the photoelectric conversion efficiencies of the DSSCs prepared by the purified SCNTs are slightly higher than those of the DSSCs prepared by the purified MCNTs. It is not only because of the different structures between SCNTs and MCNTs, but also for the greater carbon content in the purified SCNTs than the purified MCNTs. Therefore, the electronic transfer speed in the DSSCs prepared by the purified SCNTs is increased, thus resulting in higher conversion efficiencies. Table 1 shows the photoelectrochemical parameters of each DSSC after the counter electrodes prepared by different CNTs are respectively incorporated with $\mathrm{TiO}_{2}$ photoelectrodes in DSSCs.

\section{Conclusions}

After reviewing and discussing the above experimental results, the following conclusions can be drawn:

(1) Upon completion of the XRD analysis of purified and unpurified CNTs, it is evident that after purification of CNTs, the carbon impurities and metallic catalysts are significantly decreased, and the XRD absorption strength of purified CNTs is significantly increased.

(2) From the charts of IPCE and UV-vis of anthocyanin
Table 1 Photoelectrochemical parameters of the counter electrodes prepared by different CNTs after incorporation with $\mathrm{TiO}_{2}$ photoelectrodes in DSSCs.

\begin{tabular}{cccccc}
\hline Photoelectrode & Counter electrode & $V_{\mathrm{oc}}(\mathrm{V})$ & $\begin{array}{c}I_{\mathrm{sc}} \\
\left(\mathrm{mA} / \mathrm{cm}^{2}\right)\end{array}$ & $\begin{array}{c}\mathrm{FF} \\
(\%)\end{array}$ & $\eta(\%)$ \\
\hline $\begin{array}{c}\text { Degussa } \\
\mathrm{P} 25 \mathrm{TiO}_{2}\end{array}$ & $\begin{array}{c}\text { purified single-walled } \\
\text { CNTs }\end{array}$ & 0.59 & 2.65 & 51 & 0.672 \\
\hline $\begin{array}{c}\text { Degussa } \\
\mathrm{P} 25 \mathrm{TiO}_{2}\end{array}$ & single-walled CNTs & 0.47 & 2.41 & 38 & 0.420 \\
\hline $\begin{array}{c}\text { Degussa } \\
\mathrm{P} 25 \mathrm{TiO}_{2}\end{array}$ & $\begin{array}{c}\text { purified Multi-walled } \\
\text { CNTs }\end{array}$ & 0.48 & 3.12 & 39 & 0.524 \\
\hline $\begin{array}{c}\text { Degussa } \\
\mathrm{P} 25 \mathrm{TiO}_{2}\end{array}$ & Multi-walled CNTs & 0.51 & 1.71 & 44 & 0.389 \\
\hline
\end{tabular}

dye, it is apparent that the incident photon conversion trend is related to the absorption spectrum property of the dye. The IPCE and UV-vis absorption spectrums of anthocyanin dye show the same trend.

(3) $\beta$-carotene, a pigment molecule without sensitization properties, can release energy to the electrons being captured by $\mathrm{TiO}_{2}$ surface traps, thus making these electrons return to the conduction band again to continue conduction, and lengthening the electronic life cycle of the anthocyanin dye.

(4) As revealed by the experiments, after comparison of the photoelectric conversion efficiencies among the counter electrode thin films of purified SCNTs, SCNTs, purified MCNTs and MCNTs, the purified SCNT counter electrode thin film has the optimal photoelectric conversion efficiency at $0.672 \%$, which is higher than the photoelectric conversion efficiency of $0.524 \%$ obtained by purified MCNT counter electrode thin film.

\section{Acknowledgement}

This study was supported by the National Science Council of Taiwan, Republic of China under the project grant: NSC 98-2221-E-027-84.

\section{REFERENCES}

1) M. Gratzel: J. Photochem. Photobiol. C 4 (2003) 145-153.

2) M. Grätzel: Inorg. Chem. 44 (2005) 6841-6851.

3) B. O'Regan and M. Grätzel: Nature 353 (1991) 737-740.

4) A. Kay and M. Grätzel: Sol. Eng. Mater. Sol. Cells 44 (1996) 99-117.

5) A. Hauch and A. Georg: Electrochem. Acta 46 (2001) 3457-3466.

6) T. N. Murakami, S. Ito, Q. Wang, M. K. Nazeeruddin, T. Bessho, I. Cesar, P. Liska, R. H. Baker, P. Comte, P. Péchy and M. Grätzel: J. Electrochem. Soc. 153 (2006) A2255-A2261.

7) H. Zhu, H. Zeng, V. Subramanian, C. Masarapu, K. H. Hung and B. Wei: Nanotechnology 19 (2008) 465204-1-5.

8) J. Q. Wei: Nano Lett. 7 (2007) 2317-2321

9) J. Landi, R. P. Raffaelle, S. L. Castro and S. G. Bailey: Prog. Photovol. Res. Applicat. 13 (2005) 165-172.

10) K. Tennakone, G. R. A. Kumara, A. R. Kumarasinghe, P. M. Sirimanne and K. G. U. Wijayantha: Photochem, Photobiol. A 94 (1996) 217-220.

11) A. S. Polo and N. Y. Iha: Sol. Eng. Mater. Sol. Cells 90 (2006) 19361944.

12) C. G. Garcia, A. S. Polo and N. Y. Iha: Photochem. Photobio. A 160 (2003) 87-91.

13) G. P. Smestad: Sol. Eng. Mater. Sol. Cells 55 (1998) 157-178.

14) S. Hao, J. Wu, Y. Huang and J. Lin: Sol. Eng. 80 (2006) 209-214. 